\title{
OB stellar associations in the Large Magellanic Cloud: Survey of young stellar systems
}

\author{
D. Gouliermis ${ }^{1}$, M. Kontizas ${ }^{2}$, E. Kontizas ${ }^{3}$, and R. Korakitis ${ }^{4}$ \\ 1 Sternwarte der Universität Bonn, Auf dem Hügel 71, 53121 Bonn, Germany \\ 2 Department of Astrophysics Astronomy \& Mechanics, Faculty of Physics, University of Athens, 15783 Athens, Greece \\ 3 Institute for Astronomy and Astrophysics, National Observatory of Athens, PO Box 20048, 11810 Athens, Greece \\ 4 Dionysos Satellite Observatory, National Technical University of Athens, 15780 Athens, Greece
}

Received 20 January 2003 / Accepted 25 March 2003

\begin{abstract}
The method developed by Gouliermis et al. (2000, Paper I), for the detection and classification of stellar systems in the LMC, was used for the identification of stellar associations and open clusters in the central area of the LMC. This method was applied on the stellar catalog produced from a scanned $1.2 \mathrm{~m}$ UK Schmidt Telescope Plate in $U$ with a field of view almost $6.5 \times 6.5$, centered on the Bar of this galaxy. The survey of the identified systems is presented here followed by the results of the investigation on their spatial distribution and their structural parameters, as were estimated according to our proposed methodology in Paper I. The detected open clusters and stellar associations show to form large filamentary structures, which are often connected with the loci of HI shells. The derived mean size of the stellar associations in this survey was found to agree with the average size found previously by other authors, for stellar associations in different galaxies. This common size of about $80 \mathrm{pc}$ might represent a universal scale for the star formation process, whereas the parameter correlations of the detected loose systems support the distinction between open clusters and stellar associations.
\end{abstract}

Key words. galaxies: individual: LMC - galaxies: star clusters - galaxies: stellar content - surveys

\section{Introduction}

The spatial distribution and the statistical study of the properties of young stellar systems in a galaxy provide useful information on its structure and its formation history. Almost all the stars are born as members of various kinds of stellar groups, stellar associations being a very interesting one (Gomez et al. 1993; Massey et al. 1995). The variety of sizes of such stellar systems suggests a hierarchy in the formation of stellar structures as was earlier noted by McKibben Nail \& Shapley (1953). In addition, the investigation of a large sample of star forming regions in nearby galaxies allows us to study the behaviour of star formation in galactic scale. Such a study has been done with use of the spatial investigation of the star formation history throughout the whole body of dwarf galaxies (e.g. Dohm-Palmer et al. 1997) and the distribution of the most active centres of recent star formation in distant galaxies (e.g. Hunter et al. 1998).

The Large Magellanic Cloud (LMC) shows to be an ideal laboratory for the study of different stellar populations, due to its wide variety of stellar systems, it is relatively close to us (Madore \& Freedman 1998), there is low dust extinction (Harris et al. 1997) and we observe it almost face on (Westerlund 1997), while its depth seems to be very small

Send offprint requests to: D. Gouliermis,

e-mail: dgoulier@astro.uni-bonn.de/horus@mailbox.gr
(Caldwell \& Couslon 1986). In the LMC the star formation mechanisms in galactic scale have been investigated through the spatial distribution of Cepheids in this galaxy by Elmegreen $\&$ Efremov (1996). This investigation was focused on a time scale of recent star formation $\lesssim 5 \times 10^{8}$ yr. Harris \& Zaritsky (1999), expanding the research of stellar formation to larger areas and toward earlier times, give a quantitative description of the distribution of different stellar populations in the LMC. They found indications of hierarchy in the formation of young stellar groups for length-scales between about 30 and $550 \mathrm{pc}$. As far as the initial spatial distribution of newly born stellar populations and their evolution concerns, it seems that these populations are mostly confined in small scale structures as was recently found in the SMC by Maragoudaki et al. (2001).

In order to investigate the spatial distribution of the clustered young populations in a galaxy one must identify the most recent formed stellar systems. Stellar associations being typically loose stellar systems (Blaauw 1964) characterised by their bright blue populations, are considered tracers of the distribution of the youngest population in a galaxy in a specific galactic scale of about 80 pc (Efremov \& Elmegreen 1998). Still, their detection is not a trivial task. According to Hodge (1986) the differences of the samples of stellar associations in various galaxies arise from the use of different observational material and selection criteria. He concludes that with only the integrated colour and patchiness of the images as criteria the 
danger of having severe selection effects in the sample is acute and he notes that the problem of identifying stellar associations must be approached by carrying out proper controls and statistical techniques in order to be sure that we are dealing with physical groupings. Following these advises we developed an objective method for the detection of loose young stellar systems in the LMC (Gouliermis et al. 2000, from here on Paper I). The method is based on the one proposed by Kontizas et al. (1994) and the selection criteria presented by Kontizas et al. (1999).

In this paper we present the results of our investigation on the detection of loose stellar systems, in the total area of about $6.5 \times 6.5$ around the Bar of the LMC. This area according to Westerlund (1997) covers a subsystem, characterised by young stellar populations (see also Nikolaev \& Weinberg 2000). Our investigation here consists of the following steps: (1) Detection of stellar systems in the whole area of a $1.2 \mathrm{~m} \mathrm{UK} \mathrm{Schmidt}$ Telescope plate in $U$ by applying the method and the criteria of Paper I (see Sect. 2). (2) Estimation of the structural parameters of the detected systems according to the assumptions of Paper I (Sect. 3.1). (3) Classification of the systems according to their stellar density into bound, intermediate and unbound systems (Sect. 3.2), following the classification scheme proposed in Paper I. (4) In Sect. 3.3, comparison of our survey with other catalogs and objects found in the same areas. (5) Study of the spatial distribution of the detected unbound and intermediate systems in the LMC, which takes place in Sect. 4. (6) Statistical study of the properties of these systems (Sect. 5). General conclusions are given in Sect. 6.

\section{Detection of stellar systems}

\subsection{Plate description}

We applied the detection method on the whole stellar catalog of a photographic direct plate taken with the $1.2 \mathrm{~m} \mathrm{UK} \mathrm{Schmidt}$ Telescope in $U$. The ID of the plate is U12346 and its centre coordinates are $05^{\mathrm{h}} 25^{\mathrm{m}} 36^{\mathrm{s}},-69^{\circ} 35^{\prime} 30^{\prime \prime}$ (in J2000) with a field of view of about $6.5 \times 6.5$. It was exposed for $150 \mathrm{~min}$ on January 17, 1988. A UG1 filter was used on a hypersensitized Kodak IIIa- $J$ emulsion. The stellar catalog was produced by the Automated Plate Measuring Facility (APM) in Cambridge. The data were calibrated and checked for completeness (see Paper I). This plate covers the central area of the galaxy around the Bar, where most of the associations found by Lucke \& Hodge (1970) (from here on LH) are located. In addition it covers a large area southern of the LH survey, where many new systems were identified.

\subsection{Application of the method}

The method is based on star counts on a square grid, as we describe in Sect. 3 of Paper I. We repeat the star counts six times, each for a different range of magnitudes (magnitude slice), in which the stellar catalog was divided and one time for all stars brighter than $\simeq 17$ mag (cumulative catalog). The adopted magnitude slices, as well as representative values of the mean background surface stellar density and its standard deviation $\sigma$ for different areas of the plate, are listed in Table 2 of Paper I. We applied the star counts procedure on 52 different quadrilateral areas with dimensions varying from 0.3 to $2^{\circ} .0$, which cover the whole region of the plate. The selection of the boundaries of these areas was made so that each one has a uniform distribution of field stars, meaning a more or less uniform background surface density. For each area we used the corresponding mean background density and its standard deviation $(\sigma)$ in order to reveal the stellar concentrations which appear with a density exceeding the background by a specific limit in $\sigma$ s (density threshold) on the cumulative catalog, satisfying the first criterion set in Paper I (Sect. 3). According to the second criterion each concentration should appear in at least two magnitude slices, one of them being for bright stars, at the same position as in the cumulative catalog.

The method depends on two parameters. The first is the resolution limit, which corresponds to the grid element size for counting stars and sets the minimum size of the detected systems. The second is the lower density difference (in $\sigma \mathrm{s}$ ) of each low-density grid element from its neighbours so that it can be accepted as "member" of the concentration, in order different neighbouring systems lying within the same density boundaries to be separated during the detection. More details are given in Sect. 3.1 of Paper I. The method's performance concerning these parameters, as well as for different density thresholds was thoroughly checked in Sect. 3.2 of Paper I. As a consequence the grid element size was selected to correspond to $20 \mathrm{pc} \times 20 \mathrm{pc}$. The lower density difference of neighbouring grid elements in order to be accepted as members of the same concentration was set to $1 \sigma$ and the lower density of the grid elements, which represent statistical important stellar groups (density threshold) was set to $3 \sigma$, both above the local background surface density.

\section{The detected systems}

\subsection{Parameters estimation}

Structural parameters for the detected systems were estimated following the calculations and assumptions of Sect. 4.1 of Paper I. The size of each system was defined as the maximum dimension of a quadrilateral enclosing its $3 \sigma$ density boundaries. The Spitzer radius $r_{\mathrm{Sp}}$ was computed as the mean square of the stars' distances from the point of peak density within this quadrilateral. The average central stellar density of each system (in $M_{\odot} \mathrm{pc}^{-3}$ ) was estimated within the radius where half the number of its stars is contained, which may represent very well the half-mass radius of the system. The mass estimation of the systems needed a more thorough investigation and could only by achieved based on several assumptions. The difficulties in such estimation and the assumptions used are discussed in detail in Paper I. In general, in order to estimate the total mass of each detected system we had to transform the luminosities of the stars from our catalog into masses by using isochrone models from Alongi et al. (1993) and to extrapolate the number of the brighter stars to the lower mass end due to incompleteness of the data. It was assumed that all detected systems are young (with ages around $5 \mathrm{Myr}$ ) and that their Mass Functions 
Table 1. Sample table of data and estimated parameters available for the detected intermediate and unbound stellar systems. A part of the catalog of the unbound systems is shown here. The full catalogs are available at the CDS via anonymous ftp to cdsarc.u-strasbg.fr (130.79.128.5) or via http://cdsweb.u-strasbg.fr/cgi-bin/qcat?J/A+A/405/111.

\begin{tabular}{|c|c|c|c|c|c|c|c|c|}
\hline ID & \multicolumn{2}{|c|}{ (J2000) } & $\begin{array}{l}\text { Size } \\
(\mathrm{pc})\end{array}$ & $\overline{N_{\star}}$ & $\begin{array}{l}r_{\mathrm{Sp}} \\
(\mathrm{pc})\end{array}$ & $\begin{array}{c}r_{\mathrm{h}} \\
(\mathrm{pc})\end{array}$ & $\begin{array}{c}\text { Mass } \\
\left(\times 10^{3} M_{\odot}\right)\end{array}$ & $\begin{array}{c}\rho \\
\left(M_{\odot} \mathrm{pc}^{-3}\right)\end{array}$ \\
\hline A015 & 0522.0 & -7140 & 83 & $57 \pm 8$ & 26.1 & 24.9 & $1.8-3.5$ & $0.05 \pm 0.02$ \\
\hline A016 & 0520.5 & -7151 & 83 & $116 \pm 11$ & 33.0 & 30.7 & $3.4-6.9$ & $0.05 \pm 0.02$ \\
\hline A017 & 0522.8 & -7148 & 125 & $166 \pm 13$ & 60.2 & 50.6 & $4.9-9.8$ & $0.01 \pm 0.01$ \\
\hline A018 & 0522.9 & -7120 & 63 & $36 \pm 6$ & 22.8 & 19.9 & $1.1-2.3$ & $0.06 \pm 0.03$ \\
\hline A019 & 0521.1 & -7142 & 83 & $65 \pm 8$ & 30.0 & 27.0 & $2.0-4.0$ & $0.04 \pm 0.02$ \\
\hline A020 & 0516.0 & -7147 & 42 & $11 \pm 3$ & 12.1 & 12.3 & $0.4-0.8$ & $0.10 \pm 0.05$ \\
\hline A021 & 0518.4 & -7202 & 42 & $24 \pm 5$ & 21.0 & 21.4 & $0.8-1.6$ & $0.03 \pm 0.02$ \\
\hline A022 & 0523.8 & -7207 & 63 & $51 \pm 7$ & 37.7 & 40.5 & $1.6-3.2$ & $0.01 \pm 0.00$ \\
\hline A023 & 0522.8 & -7135 & 63 & $18 \pm 4$ & 20.9 & 16.6 & $0.6-1.2$ & $0.06 \pm 0.03$ \\
\hline A024 & 0513.9 & -7137 & 63 & $38 \pm 6$ & 28.9 & 25.5 & $1.2-2.4$ & $0.03 \pm 0.01$ \\
\hline A025 & 0521.9 & -7151 & 63 & $29 \pm 5$ & 27.3 & 30.2 & $0.9-1.9$ & $0.01 \pm 0.01$ \\
\hline A026 & 0501.6 & -7143 & 145 & $208 \pm 14$ & 58.7 & 53.6 & $6.1-12.2$ & $0.01 \pm 0.01$ \\
\hline A027 & 0504.3 & -7146 & 187 & $302 \pm 17$ & 66.6 & 61.4 & $8.7-17.5$ & $0.01 \pm 0.01$ \\
\hline A028 & 0502.9 & -7145 & 62 & $47 \pm 7$ & 25.9 & 24.2 & $1.5-2.9$ & $0.04 \pm 0.02$ \\
\hline A029 & 0506.4 & -7153 & 187 & $271 \pm 16$ & 72.9 & 68.1 & $7.8-15.7$ & $0.01 \pm 0.00$ \\
\hline A030 & 0505.2 & -7146 & 166 & $289 \pm 17$ & 61.5 & 53.9 & $8.3-16.7$ & $0.02 \pm 0.01$ \\
\hline A031 & 0509.6 & -7145 & 125 & $75 \pm 9$ & 53.9 & 44.5 & $2.3-4.6$ & $0.01 \pm 0.00$ \\
\hline $\mathrm{A} 032$ & 0512.6 & -7157 & 145 & $177 \pm 13$ & 66.5 & 62.4 & $5.2-10.4$ & $0.01 \pm 0.00$ \\
\hline A033 & 0510.8 & -7146 & 83 & $39 \pm 6$ & 36.0 & 26.2 & $1.2-2.5$ & $0.03 \pm 0.01$ \\
\hline A034 & 0509.8 & -7151 & 125 & $92 \pm 10$ & 48.6 & 35.2 & $2.8-5.5$ & $0.02 \pm 0.01$ \\
\hline A035 & 0507.6 & -7155 & 125 & $119 \pm 11$ & 67.8 & 62.0 & $3.5-7.1$ & $0.01 \pm 0.00$ \\
\hline
\end{tabular}

have slopes varying from $\Gamma \simeq-1.0$ to -1.6 . Considering that the estimated masses depend on the errors of counting stars and mainly on the span of the adopted MF slopes and age, the extracted estimation errors can be as large as an order of a magnitude.

\subsection{Classification of the detected systems}

We used the central stellar density of each system $(\rho)$ in order to classify it as bound $\left(\rho \geq 1.0 M_{\odot} \mathrm{pc}^{-3}\right)$, intermediate $\left(0.1<\rho<1.0 M_{\odot} \mathrm{pc}^{-3}\right)$ or unbound system $\left(\rho \leq 0.1 M_{\odot} \mathrm{pc}^{-3}\right)$. This classification scheme is proposed in Sect. 4.1 of Paper I, where a discussion on the stability of stellar systems according to these density limits is also presented. In total 494 stellar systems were found in the area of $6.5 \times 6.5$ around the LMC Bar in the $U$ plate. From them 82 were classified as bound, 259 as intermediate and 153 as unbound systems. Our interest here is focused on the intermediate and unbound detected systems. They represent loose young stellar concentrations, which our method was designed for. Consequently almost all the detected bound systems were found with sizes on the detection limit of $20 \mathrm{pc}$ and they are of no particular importance in this investigation. The detected intermediate and unbound systems and their parameters are available in two tables from CDS (one table for every category). Table 1 here is a sample of the data given in these tables. In Col. 1 the identification number of each system is given. Columns 2 and 3 show the coordinates of the dynamical centre of the system, which is taken to be the most dense part of the system. The size of each system is given in Col. 4, and in Cols. 5-7 we present the number of bright stars $N_{\star}$ counted within the system's boundaries, its Spitzer radius $r_{\mathrm{Sp}}$, as well as its half-mass radius $r_{\mathrm{h}}$. The mass limits and the mean central stellar density of each system is given in Cols. 8 and 9 respectively. The identification numbers of the intermediate systems have the prefix "O", since the large majority of these systems most probably represents Open Clusters. For the unbound systems, considering that they are mostly Stellar Associations we used the prefix "A". This numbering scheme will be used from here on.

During the investigation on the detected systems in two selected areas of $U$ plate, as was presented in Paper I, it was found that according to the spectral classification, all the identified unbound systems show to have the characteristics of OB stellar associations. Considering the various uncertainties in the estimation of the parameters of the systems, we also found that at least $30 \%$ of the intermediate systems are probably stellar associations, since they show to have stellar densities very close to the limit of $\rho \simeq 0.1 M_{\odot} \mathrm{pc}^{-3}$, while $\sim 68 \%$ of them showed an excess of bright $\mathrm{OB}$ stars and we accepted them as candidate stellar associations or open clusters. Taking these under consideration we present the results of our investigation on the detected intermediate and unbound systems in the whole $U$ plate. The map of the survey of these systems is shown in Fig. 1.

\subsection{Comparison with other surveys}

In this section we present the results of the comparison of our survey with other already published catalogs of stellar systems in the LMC. This comparison is made in order to test the efficiency of our method in comparison with others, concerning the number of the detected systems, their positions and their estimated sizes. We state in the introduction, that the results of the detection of a specific type of stellar systems is directly connected to the method and data used. Thus, the detection 


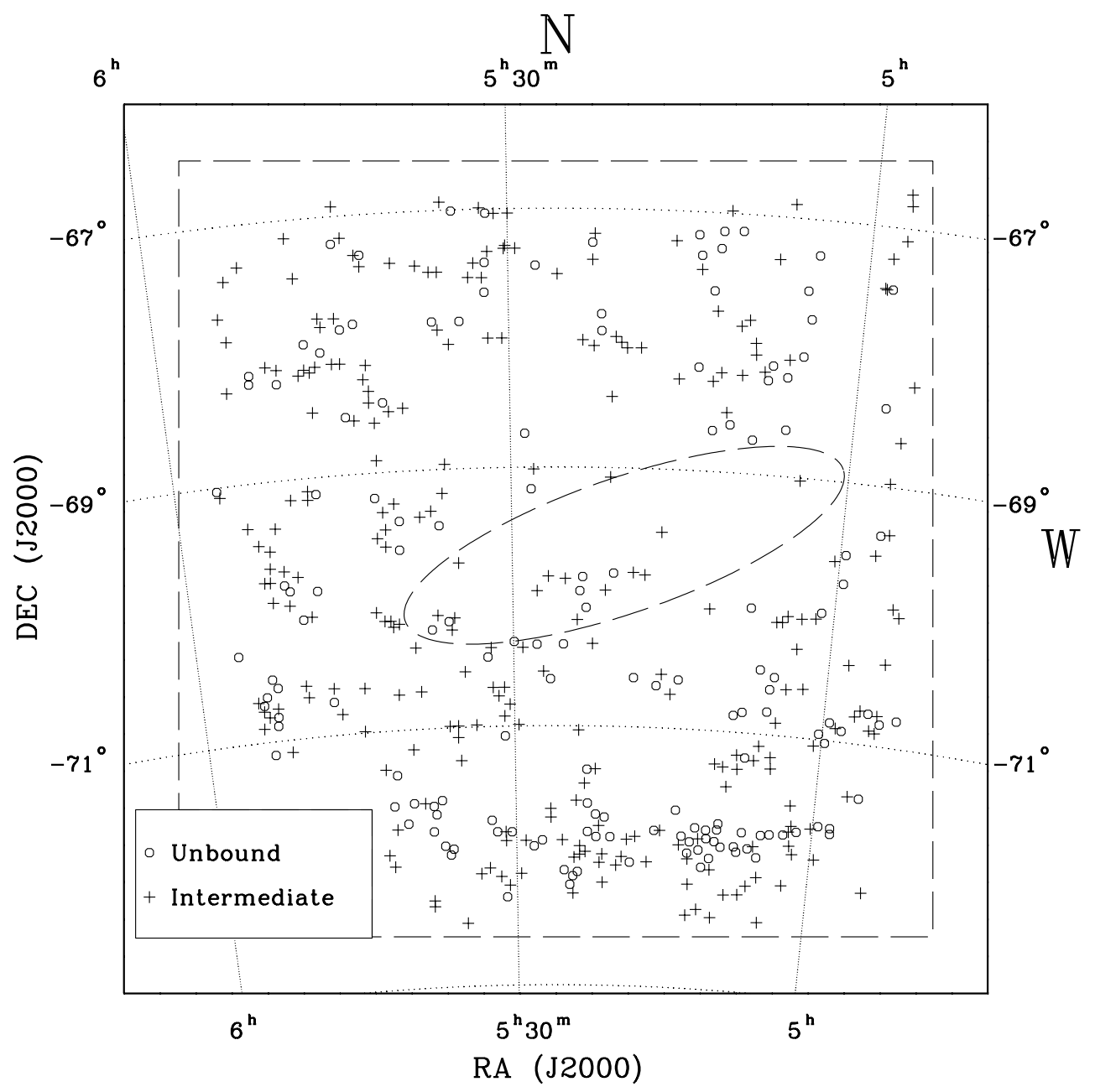

Fig. 1. Survey of the intermediate and unbound systems detected in $U$ in the area of $6.5 \times 6.5$ around the LMC Bar. The points represent the densest centres of the systems. The limits of the plate and the area of the Bar are marked with dashed lines.

criteria adopted are very important, if we are to compare the results of two different detection methods. The most complete available catalog of stellar systems in the LMC is presented by Bica et al. (1999). This survey covers the whole area of the galaxy and contains almost 6500 clusters, associations and nebulae. Still we are not going to compare our results with those of Bica et al., due to our differences with these authors concerning the definition of a stellar association and in consequence the adopted criteria. We found though all the Bica et al. (1999) objects related to our systems as is shown later.

The method of Bica et al. (1999) as was presented by Bica \& Schmitt (1995) is based on identification by eye on ESO/SERC $R$ and $J$ Sky Survey Schmidt films. In addition the distinction between clusters and associations according to these authors "is based primarily on the stellar density, but additional criteria are the magnitude distribution of stars and the occurrence of irregular shape, which characterise associations" (Bica \& Schmitt 1995). It seems that the detection criteria used was purely qualitative. Consequently systems classified by these authors as associations do not meet systematically the criteria, which characterise this type of systems (see e.g. Kontizas et al. 1999). On the other hand the definition of stellar associations (roughly as loose concentrations of bright blue stars) used by
Lucke \& Hodge (1970) is in line with the typical criteria for stellar associations and the criteria of our method. Thus we compare our results here with the LH catalog of LMC associations due to the similarities of the two methods, as far as the criteria concerns.

In the area covered by the $U$ plate there are $102 \mathrm{LH}$ associations (Lucke \& Hodge 1970). We checked the coincidence of our identified systems with the LH associations for each smaller area, on which we applied the detection method. For each area we constructed the cumulative isodensity contour map (like the maps of Fig. 4 in Paper I), so that the limits of each detected system to be drawn after the limits of the LH associations of the same area were overplotted. The comparison was carried out by eye on these maps. We applied this kind of comparison due to the fact that the systems in both surveys have not symmetrical appearance, so it is possible that a centre to centre comparison wouldn't give any useful results. We accepted that two systems coincide even when these systems overlap each other with no centre coincidence. There are also cases where one system in the one catalog overlaps more than one systems of the other.

We identified 73 LH associations. The 29 unidentified LH associations are located in crowded regions characterised by 
high nebulosity, which makes their identification a rather difficult task. Among them are the easily recognized but highly nebulous LH 58 (Garmany et al. 1994) and the LH associations located near 30 Doradus (Parker 1993; Parker \& Garmany 1993). More specifically, five of the LH associations not detected by us are located within and around the area of 30 Doradus (LH 81, LH 90, LH 100, LH 105 \& LH 113), while additional seven are located in the dense area northwest of and outside the Bar (LH 58, LH 67, LH 71, LH 73, LH 74 \& LH 85). In addition thirteen of the undetected LH associations belong to the group of the 19 LH stellar associations, which cover the central area and the northwest edge of the Bar. From these we detected LH 11, LH 12, LH 20, LH 39, LH 44 \& LH 59, which are all located very close to the limits of the Bar. Finally our method did not detect four small LH associations: LH 5, LH 82, LH 88 and LH 122. We conclude, thus, that the method did not perform well in crowded regions and in regions where nebulosity is very high. This result is not due to the detection method itself, but due to the nature of the data used. It is worth noting that in the $U$ filter dense nebula regions like the 30 Doradus and the Bar are shown to be extremely bright. Consequently, the detection of stars by the scanning machines is affected by this phenomenon and the resulting stellar catalogs in these regions are incomplete. Indeed in the areas covering the Bar, where we performed our method, we noticed phenomena of bad statistics. The $U$ plate on the other hand is one of the most appropriate for the detection of young stellar populations, which is the aim of this survey. In addition the coincidence of our detected systems with LH associations in the rest areas of the plate was excellent.

In general we were able to detect 412 intermediate and unbound systems in the same area where Lucke \& Hodge detected 102 associations, meaning that this survey is almost four times richer than the one by Lucke \& Hodge. Although the latter relied upon various criteria in addition to star density, the central criterion of both methods is the appearance of stellar associations as loose concentrations of bright young stars. So, one may ask why this difference between the two surveys. Considering that stellar density plays important role in both methods it seems that the higher number of systems in our survey is probably due to the ability of our method to detect loose stellar concentrations in areas where the eye is not able to identify any significant stellar group unless there is nebulosity related.

We confirmed the results of the comparison of the positions of the systems in our survey with those of the LH associations using $\operatorname{SIMBAD}^{1}$ (http://simbad.u-strasbg.fr/). We searched for all the known stellar systems (as well as for other objects, as HII regions, nebulae, etc.), which are located around the centre of each one of our systems within a search radius equal to the half of the size of the system as it was estimated in Sect. 3.1. It should be taken under consideration that this kind of "centre to centre" search, within specific search radii, gave excellent results as far as the coincidence of unbound systems and LH associations concerns, verifying the results of our search. On the other hand, the search in SIMBAD

1 SIMBAD: SET OF IDENTIFICATIONS, MEASUREMENTS AND BIBLIOGRAPHY FOR ASTRONOMICAL DATA.

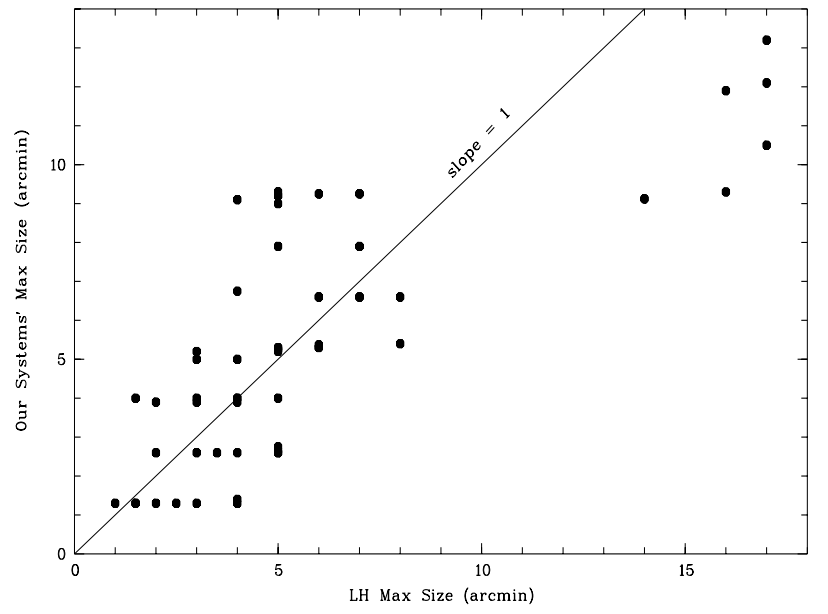

Fig. 2. Comparison of the maximum sizes of the systems found in both our and LH surveys.

for coincidence of known objects with the detected intermediate systems verified only two thirds of the coincidence of intermediate systems with LH associations found by us, probably due to the systematically smaller search radii, which were used in SIMBAD for these systems. As it is said earlier, there are cases, where more than one of our systems coincide with a LH association. Probably these LH associations are stellar aggregates, for which we detected the various internal subsystems. There are also cases, where more than one LH associations were found to coincide with one of the systems of our survey. Such a case is the one of LH 93, LH 94, LH 97 \& LH 98 west of 30 Doradus, which Lucke \& Hodge (1970) mark within the limits of one larger association (LH 96), and which we detected through two systems (A110 and O168), one of them being a large stellar association.

In Fig. 2 we compare the maximum sizes of our systems with the ones of the corresponding LH associations. In cases where more than one systems of one catalog coincide with only one of the other, we compared the size of this system with the total size of all the subsystems coinciding with it. As is shown from Fig. 2 the coincidence is very good, considering a gap for sizes between $\sim 10$ and $15 \mathrm{arcmin}$. This gap is due to the lack of systems with sizes in this range in the LH catalog only. The two groups of systems shown in Fig. 2 give a linear correlation with slopes $0.9_{-0.2}^{+0.1}$ for the small systems $(<10$ arcmin $)$ and $0.95_{-0.5}^{+.05}$ for the larger ones ( $>10 \mathrm{arcmin})$. These values give a very good coincidence of our systems' sizes with the ones of LH associations, being very close to the value of excellent coincidence (slope $=1$ ) also shown in Fig. 2. In the case of LH associations with sizes less that 10 arcmin the correlation show to follow this line, considering the relatively wide spread in sizes. In the case of the larger systems, though the slope is nearly unit, the zero point of the fitted line is well above zero, moved by about 5 arcmin. This implies that the "larger" systems as was detected by us are systematically smaller that the corresponding LH associations by about 5 arcmin. Probably this is due to two factors: (1) The sizes as were estimated here are based purely on star counts and we did not consider the related nebulosity, as Lucke \& Hodge did. (2) The correspondence of the systems 
Table 2. Nomenclature, acronyms and references on the surveys of various kinds of objects found to be connected to our survey according to SIMBAD.

\begin{tabular}{|c|c|c|c|c|}
\hline $\begin{array}{l}\text { Object } \\
\text { Type }\end{array}$ & $\begin{array}{l}\text { SIMBAD } \\
\text { Nomenclature }\end{array}$ & Acronyms & References & $\begin{array}{c}\text { Number of } \\
\text { objects }\end{array}$ \\
\hline \multirow[t]{2}{*}{ Globular Cluster } & $\mathrm{GlC}$ & NGC & Sulentic \& Tifft (1973) & 1 \\
\hline & & H88 & Hodge (1988) & 2 \\
\hline \multirow[t]{11}{*}{ Cluster of Stars } & $\mathrm{Cl}^{*}$ & SL & Shapley \& Lindsay (1963) & 105 \\
\hline & & NGC & Sulentic \& Tifft (1973) & 53 \\
\hline & & LT & Lortet \& Testor (1984) & 1 \\
\hline & & H88 & Hodge (1988) & 19 \\
\hline & & [BH88] & Bhatia \& Hatzidimitriou (1988) & 8 \\
\hline & & BMG & Bhatia \& MacGillivray (1989) & 1 \\
\hline & & КMHK & Kontizas et al. (1990) & 87 \\
\hline & & BRHT & Bhatia et al. (1991) & 10 \\
\hline & & ZHT AN & Zaritsky et al. (1997) & 3 \\
\hline & & [BE99] & Battinelli \& Efremov (1999) & 4 \\
\hline & & BSDL & Bica et al. (1999) & 50 \\
\hline \multirow[t]{4}{*}{ Open (galactic) Cluster } & $\mathrm{OpC}$ & HS & Hodge \& Sexton (1966) & 20 \\
\hline & & NGC & Sulentic \& Tifft (1973) & 3 \\
\hline & & BMG & Bhatia \& MacGillivray (1989) & 17 \\
\hline & & BRHT & Bhatia et al. (1991) & 4 \\
\hline \multirow[t]{5}{*}{ Association of Stars } & $\mathrm{As}^{*}$ & LH & Lucke \& Hodge (1970) & 60 \\
\hline & & NGC & Sulentic \& Tifft (1973) & 17 \\
\hline & & BMG & Bhatia \& MacGillivray (1989) & 1 \\
\hline & & KKDAB & Kontizas et al. (1994) & 1 \\
\hline & & BSDL & Bica et al. (1999) & 219 \\
\hline \multirow[t]{5}{*}{ HII (ionized) region } & HII & LHA $120-\mathrm{N}$ & Henize (1956) & 28 \\
\hline & & PKS & Shimmins \& Day (1968) & 1 \\
\hline & & NGC & Sulentic \& Tifft (1973) & 2 \\
\hline & & DEM L & Davies et al. (1976) & 60 \\
\hline & & FHW95 & Filipovic et al. (1995) & 1 \\
\hline \multirow[t]{2}{*}{ Nebula of unknown nature } & $\mathrm{Neb}$ & NGC & Sulentic \& Tifft (1973) & 2 \\
\hline & & BSDL & Bica et al. (1999) & 67 \\
\hline Super-giant shell & & SGsh-LMC & Meaburn (1980) & 2 \\
\hline Star Forming Region & & Shapley- & Shapley (1956) & 6 \\
\hline \multirow[t]{4}{*}{ Molecular Cloud } & $\mathrm{MoC}$ & $\mathrm{LMC}-\mathrm{CO}$ & Morgan (1992) & 2 \\
\hline & & [CK96] & Caldwell \& Kutner (1996) & 2 \\
\hline & & [KRB97] & Kutner et al. (1997) & 2 \\
\hline & & [JGB98] & Johansson et al. (1998) & 4 \\
\hline
\end{tabular}

is not always one to one, since as we already stated there are cases where there are more than one systems of our catalog corresponding to one system in the $\mathrm{LH}$ catalog and vice versa. So, the comparison of the overall size of all these systems to the size of the corresponding one can not be precise.

As a second step to our search for objects related to our systems through SIMBAD we used the catalogs by Lucke \& Hodge (1970) and Bica et al. (1999) to find additional "interrelations" between these objects, as well as to find new objects. In Table 2 we present summarised results of our SIMBAD search. We found 9 different types of non-stellar objects given in Col. 1 of the table. The nomenclature and acronyms of these types are given in Cols. 2 and 3 respectively. In Cols. 4 and
5 we give the corresponding bibliographical references and the number of objects found per acronym. Maybe the most interesting results are the ones concerning clusters, associations and HII regions, as they were classified by other authors: 341 clusters, 298 associations and 92 HII regions found in SIMBAD to coincide in location with the intermediate and unbound systems detected by us. Ofcourse one cannot exclude the chance of superposition of SIMBAD clusters on our associations. This can be proved from the Galactic open and globular clusters also found in SIMBAD. Still, the estimation of an accurate fraction for the cases of super-positioning is almost impossible. Anyhow, the coincidence of the loci of the systems of our survey with clusters and small associations found by other 


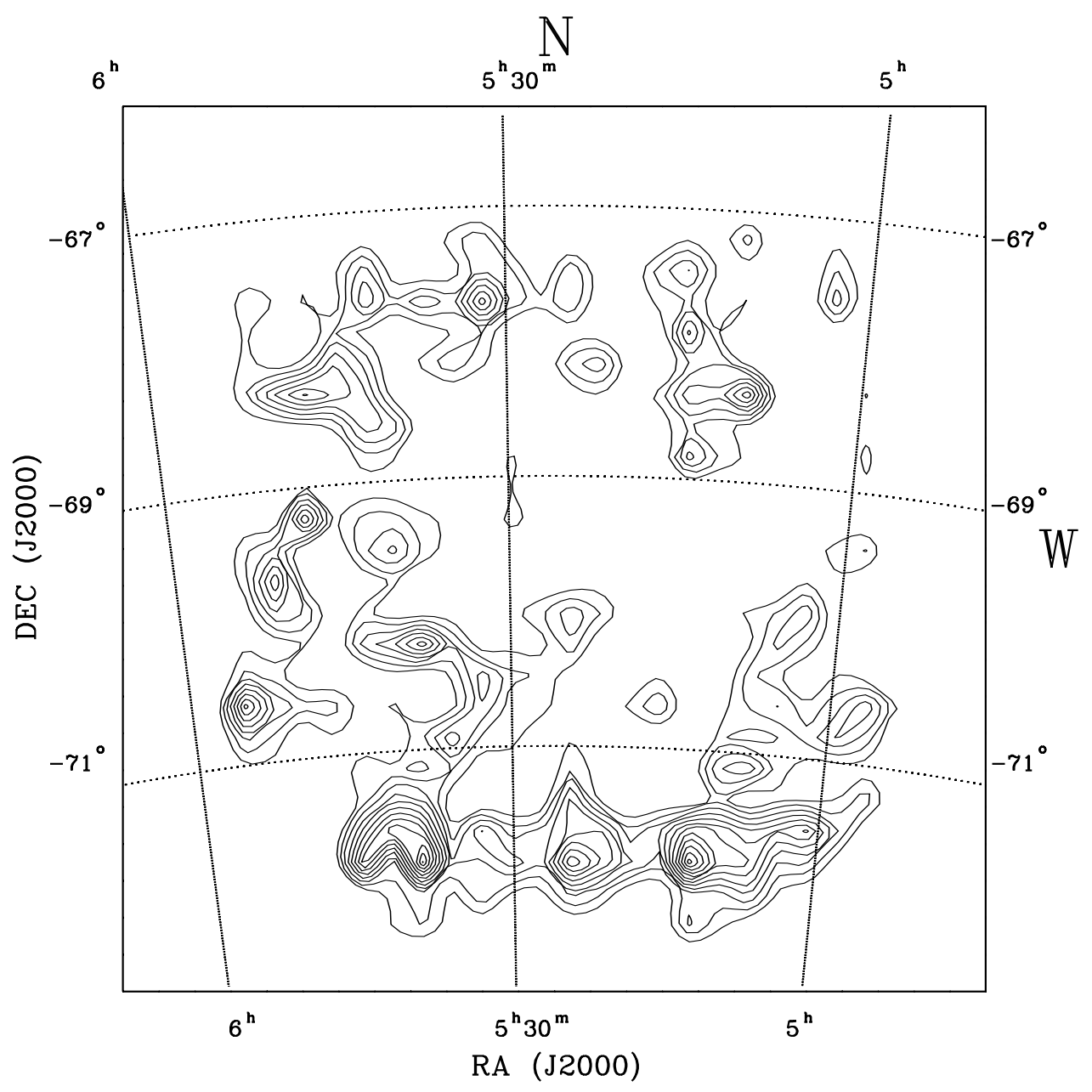

Fig. 3. Isodensity contour map produced by counting both the intermediate and unbound systems. These systems show filamentary structures (with some compact parts) in the external regions around the Bar of the LMC.

authors can be approached as a physical phenomenon, since these small systems may represent core clusters in our associations. In total 225 intermediate and unbound systems of our survey were found to be related to 818 non-galactic SIMBAD objects. We compiled a catalog of all these objects. This catalog contains the systems of our survey (Col. 1), the related SIMBAD objects and the relations between them (Cols. 2 and 3), their coordinates (Cols. 4 and 5) and the corresponding SIMBAD nomenclature (Col. 6). Table 3 shows a small portion of this catalog, which is not presented in full here due to its size and which is available upon request to the authors.

\subsection{Completeness of the survey in the bar}

In order to quantify the incompleteness of our survey in the area of the LMC Bar, we use the LH survey. Since we know the total number of $\mathrm{LH}$ associations and of our systems in the whole region, as well as in the area of the Bar, we can extrapolate the number of the systems we detected in the whole plate to the expected number of detected systems in the Bar area. In the whole area of the plate except the Bar (according to its limits as are plotted in Fig. 1) we detected 147 unbound and 246 intermediate systems. In the same area there are $85 \mathrm{LH}$ associations. If we limit our catalog in the area, which is covered by the LH survey, then we find 105 unbound and 195 intermediate systems. This suggests that our survey covers $\sim 1.2$ times more unbound and $\sim 2.5$ times more intermediate systems than the survey of LH associations. If we consider that the distribution of the systems in the area of the Bar is uniform and that the detection performs consistently in every area of the plate, since the area of the Bar includes $17 \mathrm{LH}$ associations, then we should expect to detect $\sim 21$ unbound and $\sim 39$ intermediate systems. We detected 6 unbound and 14 intermediate systems, which gives a completeness of $35 \%$ for the unbound and of $27 \%$ for the intermediate systems in the area of the Bar.

\section{Spatial distribution of the systems}

From the map of Fig. 1 one may suspect the appearance of large structures, which are formed by the systems. The incomplete detection of systems in the area of the Bar produces the hole, which is shown in this figure. The separate maps of the detected systems for each category show these structures as well. We counted the detected intermediate and unbound systems in quantrilateral grid with grid elements of sizes around $230 \mathrm{pc}$, which is the size of a typical large stellar aggregate in the LMC (e.g. Maragoudaki et al. 1998) and we produced 

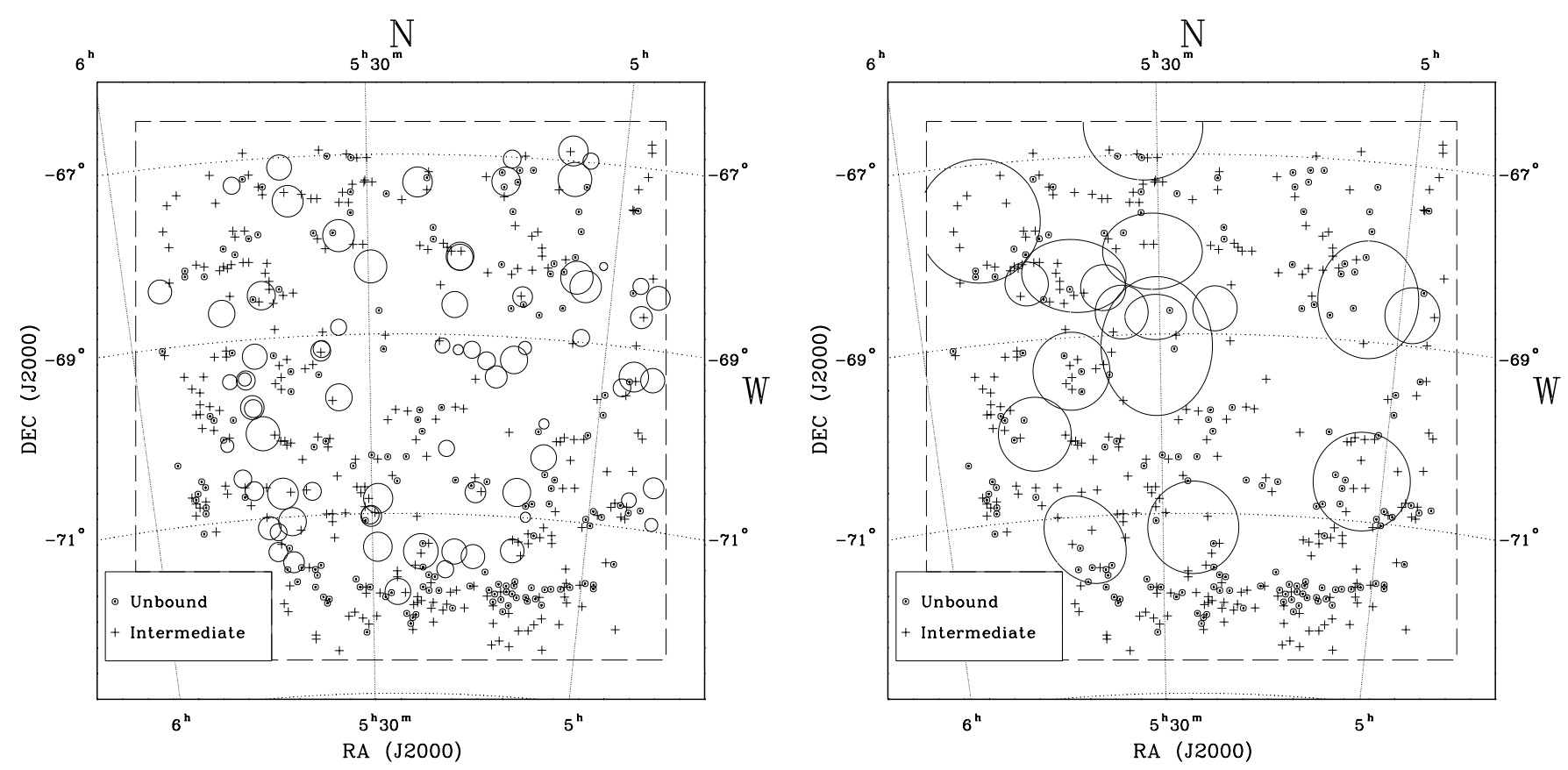

Fig. 4. The loci of the intermediate and unbound systems on those of giant (left panel) and super-giant HI shells (right panel), as found by Kim et al. (1999).

the corresponding isodensity contour map shown in Fig. 3. For the production of the map we didn't use any specific density threshold, since we used "clean" numbers of systems not contaminated by any "background" contribution.

From the isodensity contour map of Fig. 3, the existence of filamentary structures, which are formed by the detected systems is apparent. These structures show also to prefer the outer parts in the whole region. This could be due to the incomplete detection in the area of the Bar, but could also be due to the formation of the systems itself. If we consider that in the area of the Bar we detected only the $27 \%$ of the expected intermediate systems, then one should expect to see a strong concentration of these systems in the area of the Bar. Still, this concentration would leave unaffected the structures shown on the NE and SW part of the map of Fig. 3, due to the lack of systems in the areas between the Bar and these structures. This shows to be the case also for the unbound systems, of which we detected only the $35 \%$ in the area of the Bar. Very interesting in the NNE part of the isodensity contour map is the arc structure, which is located right below of the super-bubble Shapley III (LMC 4), which several authors (e.g. de Boer et al. 1998; Efremov et al. 1998) have investigated concerning its star formation history. One may ask if other similar structures, which we observe in the isodensity contour map are connected to such star forming shells.

More information on such an investigation can be given from observations on atomic hydrogen (HI) in the LMC, like the ones presented by Kim et al. (1999). These authors note that the structure of $\mathrm{HI}$ in the LMC is characterised by a large number of shells, as well as of filamentary and spiral structures. Taking under consideration that we detected stellar systems using data obtained in $U$, as well as the criteria chosen for the detection and acceptance of the systems as candidate stellar associations, then one should expect to find a relative coincidence of the spatial distribution of the systems in our cata$\log$ with the spatial distribution of atomic hydrogen, which is a good indicator of recent star formation.

Indeed such a coincidence was found, when we overplotted the map of the detected intermediate and unbound systems on the corresponding part of the HI map of LMC by Kim et al. (1999). It was rather interesting that the large filamentary structure in the SW part of our survey (see Fig. 1 or Fig. 3) found an almost perfect correspondence in the survey of Kim et al. (1999). Another interesting result of this comparison is that many of our systems are distributed around areas empty in HI (holes). Kim et al. using their observations and additional observations in $\mathrm{H} \alpha$, classified 103 candidate giantand 23 supergiant-shells. We show in Fig. 4 the comparison of the positions of the stellar systems in our survey with the edges of the giant (left panel) and supergiant (right panel) shells detected by Kim et al. This figure is an additional indication that probably some of the systems in our survey are related to the boundaries of such shells. Consequently the distribution of the systems forms arc-like structures shown in the contour map of Fig. 3. Could these systems be the result of star formation events happening on the edges of HI shells? Probably.

For example this seems to be the case for the systems found to coincide with the "conjunction" point of the supergiant shells in the NE part of the map (Fig. 4 - right panel). Stellar systems of our survey were also found projected on the inner areas of some shells. Kim et al. comment that the shells which cover HII regions and known $\mathrm{OB}$ associations seem to expand faster. This fact implies an additional input of mechanical energy from active star forming regions. Indeed we compared the areas of the HII regions found by SIMBAD to be related to systems of our survey and we found that more than half of them are 
Table 3. Sample of the catalog of objects found by SIMBAD located in areas covered by the intermediate and unbound systems of this survey. The full catalog is available upon request to the authors.

\begin{tabular}{|c|c|c|c|c|c|}
\hline \multirow[t]{2}{*}{ ID } & \multirow{2}{*}{$\begin{array}{l}\text { Simbad } \\
\text { Object }\end{array}$} & Additional Related & RA & Dec & Type \\
\hline & & Objects & \multicolumn{2}{|c|}{ (J2000) } & \\
\hline \multirow[t]{3}{*}{ A015 } & BSDL 1416 & & 052159 & -7141.3 & As* \\
\hline & BSDL 1424 & in BSDL 1428 & 052205 & -7141.9 & As* \\
\hline & BSDL 1428 & & 052208 & -7140.8 & As* \\
\hline \multirow[t]{2}{*}{ A016 } & BSDL 1304 & & 052009 & -7153.2 & As* \\
\hline & BSDL 1321 & & 052032 & -7153.4 & As* \\
\hline \multirow[t]{4}{*}{ A017 } & КМHK 846 & & 052302 & -7145.4 & $\mathrm{Cl}^{*}$ \\
\hline & BSDL 1419 & & 052159 & -7148.3 & As* \\
\hline & BSDL 1475 & & 052241 & -7148.1 & As* \\
\hline & BSDL 1532 & & 052330 & -7148.2 & $\mathrm{Cl}^{*}$ \\
\hline A018 & BSDL 1483 & in DEM L 164 & 052250 & -7121.1 & As* \\
\hline \multirow[t]{4}{*}{ A019 } & КМHK 824 & & 052131 & -7143.1 & $\mathrm{Cl}^{*}$ \\
\hline & BSDL 1354 & & 052109 & -7141.9 & As* \\
\hline & BSDL 1372 & & 052119 & -7143.9 & As* \\
\hline & BSDL 1369 & & 052120 & -7141.9 & As* \\
\hline \multirow[t]{2}{*}{ A020 } & HS 202 & = KMHK 709 & 051603 & -7148.3 & $\mathrm{OpC}$ \\
\hline & BSDL 1088 & LHA $120-\mathrm{N} 194$ in it & 051557 & -7147.8 & As* \\
\hline \multirow[t]{3}{*}{ A023 } & DEM L 165 & $=$ LHA 120-N 198 & 052227 & -7135.9 & HII \\
\hline & BSDL 1479 & in LHA 120-N 198 & 052246 & -7136.2 & As* \\
\hline & BSDL 1493 & in LHA 120-N 198 & 052256 & -7136.0 & $\mathrm{Cl}^{*}$ \\
\hline A025 & КМHK 829 & & 052146 & -7151.1 & $\mathrm{Cl}^{*}$ \\
\hline A026 & BSDL 460 & & 050148 & -7143.2 & As* \\
\hline \multirow[t]{3}{*}{ A027 } & NGC 1840 & in BSDL 586 & 050518 & -7145.7 & $\mathrm{Cl}^{*}$ \\
\hline & BSDL 586 & & 050452 & -7146.2 & As* \\
\hline & BSDL 604 & in BSDL 586 & 050523 & -7147.1 & As* \\
\hline A029 & КМHK 551 & $=$ SL 235 & 050623 & -7149.1 & $\mathrm{Cl}^{*}$ \\
\hline \multirow[t]{3}{*}{ A030 } & NGC 1840 & $=$ KMHK 529 & 050518 & -7145.7 & $\mathrm{Cl}^{*}$ \\
\hline & BSDL 586 & NGC1840 in it & 050452 & -7146.2 & As* \\
\hline & BSDL 604 & in BSDL 586 & 050523 & -7147.1 & As* \\
\hline A034 & BSDL 757 & & 050910 & -7152.4 & As* \\
\hline
\end{tabular}

located at the inner part of HI shells. It is interesting to note that the ages of the shells, as were estimated by Kim et al. (1999) are varying from about 2 to $11.5 \mathrm{Myr}$. This age estimation is in good agreement with the age of 5-10 Myr we assumed for the transformation of luminosities to masses for the detected systems during the estimation of their structural parameters.

\section{The systems' parameters}

\subsection{Sizes and masses of the systems}

The sizes of the detected systems were measured as shown in Sect. 3.1. If we take all three categories of detected systems under consideration, we see that as one moves from the category of the most compact systems (bound) toward the less compact ones (unbound), the corresponding dimensions are getting larger with the bound systems having sizes close to our detection limit and the unbound representing the larger detected systems. More specifically as far as the intermediate systems concerns most of them have sizes clustered around $30 \mathrm{pc}$, while there are some systems with sizes up to 160 pc (Fig. 5 - left panel). The size distribution of the unbound systems shows a peak at about $70 \mathrm{pc}$ and a second shorter peak at about $130 \mathrm{pc}$. Considering that the unbound systems found in Paper I are true stellar associations (as the spectral classification showed), then possibly all the unbound stellar systems of this survey represent the true population of stellar associations in the central area of LMC. The mean size of these systems was found around $85 \mathrm{pc}$. This result is in good agreement with the size distribution for stellar associations of galaxies in the Local Group, as was found by various investigators as will be shown below.

In Table 4 a compilation of existing data on detection methods and measured sizes is given for stellar associations in different galaxies. In Cols. 1 and 2 the name and the Hubble type of the galaxies are given. In Col. 3 we give the number of detected associations, while Col. 4 shows the minimum, average and maximum sizes of the detected associations. The corresponding references and methods are given in Cols. 5 and 6 respectively. This table shows the differences between galaxies as far as the number of detected associations and their sizes concerns. Hodge (1986), using a similar compilation of data available at that time, argues that the differences of the detected number of associations in various galaxies is a result of their greater distances, which makes the recognition of small associations more difficult and which leads to preferentially interpreting complexes of associations as single objects. Consequently, the associations of more distant galaxies are found to be larger.

In addition, according to the same author the differences in the properties of stellar associations in different galaxies arise 

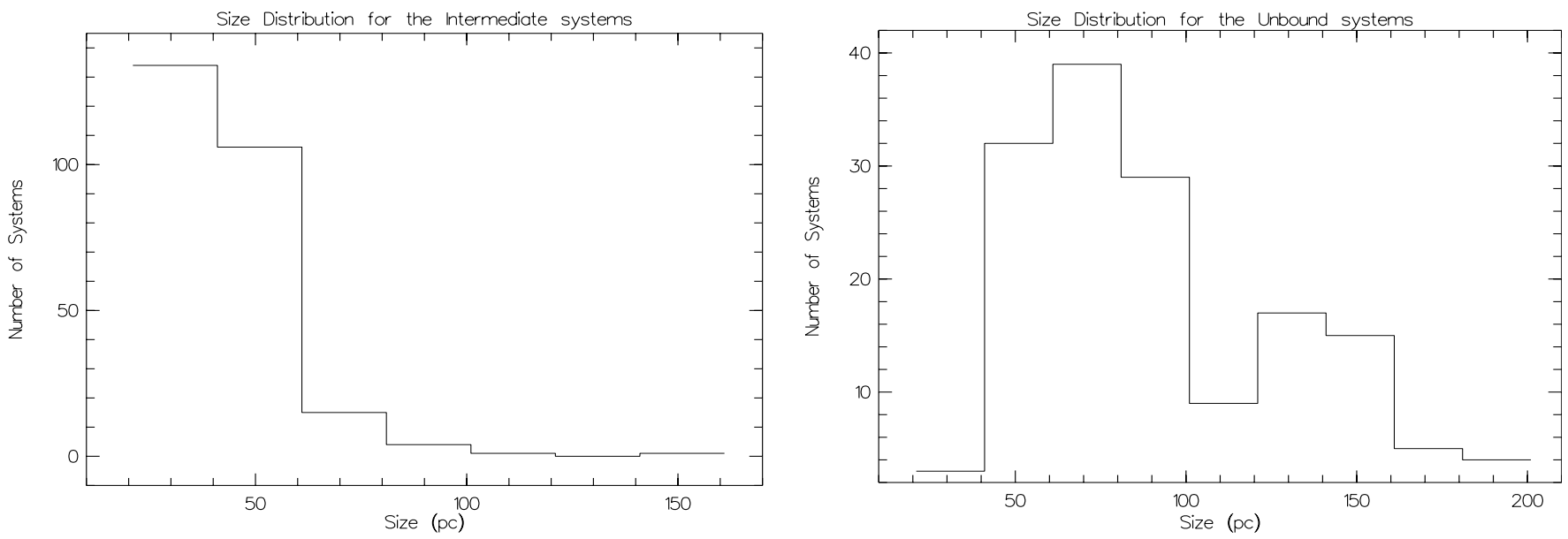

Fig. 5. Size distribution for the detected intermediate (left panel) and unbound systems (right panel).

also from the use of different observational material and detection criteria. So the different sizes of associations in different samples could be a selection effect. Efremov (1988) found that the mean size of the OB associations $(\simeq 80 \mathrm{pc})$ is a universal scale for the process of star formation in the galactic discs and Ivanov (1996) discuss the use of the average size of OB associations as a distance indicator of the parent galaxy. The same author found that the average diameter of the stellar associations of eight galaxies detected using CCD observations is $84 \pm 15 \mathrm{pc}$, very close to Efremov's value. The average size of our detected unbound systems ( $86 \mathrm{pc}$ ) seems to be in a very good agreement with both these values. From the data of Table 4 one can see that more than half of the surveys include associations with average sizes around the value of $80 \mathrm{pc}$ (between 65 and 93 pc). Almost 30\% of the surveys have associations with sizes larger than about $114 \mathrm{pc}$ up to $440 \mathrm{pc}$ and there is only a small fraction (3 surveys) with smaller limits for the sizes of stellar associations ( 18 to $46 \mathrm{pc}$ ).

If we select from Table 4 the surveys of stellar associations, which have been compiled with the use of more advanced detection techniques (not by eye) then we limit the sample of surveys of associations to half. Almost all of them are in agreement on the average size of stellar associations. Specifically all 12 except 2 surveys give the size of a stellar association to be between 65 and $93 \mathrm{pc}$; this gives an average size of stellar associations equal to $80 \pm 3 \mathrm{pc}$, in excellent agreement with the universal scale of star formation as given by Efremov (1988). Thus, one could almost safely conclude that as far as the size of stellar associations concerns, this might represent a specific global length-scale of star formation in a galaxy. Still, there are differences between different surveys of associations (like in the total number of associations in a galaxy), which might as well depend on the total luminosity or Hubble type of the host galaxy (Hodge 1986).

Concerning the mass estimation of each detected stellar system, it should be noted that the MF slope of the systems was the most important assumption and that the uncertainties of the estimated masses of up to an order of a magnitude are due to the range of the adopted MF slopes (see Sect. 4.1, Paper I). This range $(-1.6 \lesssim \Gamma \lesssim-1.0)$ is in excellent agreement with

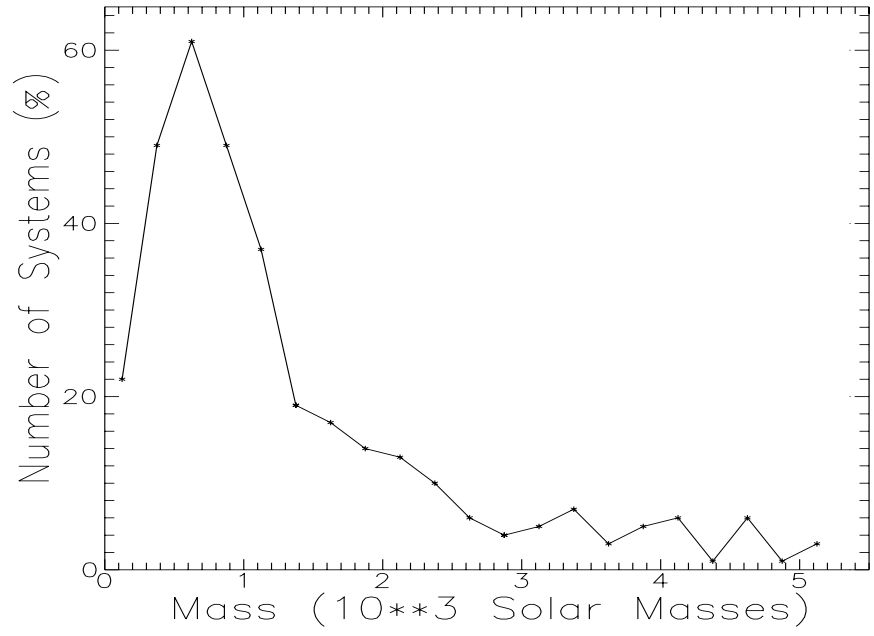

Fig. 6. Frequency distribution of the masses of the detected intermediate and unbound systems. Masses are given in $10^{3} M_{\odot}$, whereas the number of the systems are in percentage of the total detected systems.

the established IMF slopes of LH associations by several detailed studies of their stellar content (LH 117/118: Massey et al. 1989; 30 Dor area: Parker \& Garmany 1993; LH 58: Garmany et al. 1994; LH 47/48: Oey \& Massey 1995; Will et al. 1997; LH 1/2/5/8: Parker et al. 2001). These slopes were found to be comparable to each other, and clustered around $\Gamma \simeq-1.5$.

From the results of the investigations above, we were able to test the mass estimation for four of our detected systems: A129/130 (LH 47/48) and O123/A092 (LH 117/118). The MF slopes of the coinciding LH associations (LH 47/48: Oey \& Massey 1995; Will et al. 1997; LH 117/118: Massey et al. 1989) were used for an estimation of their masses in the range $0.8 \lesssim M / M_{\odot} \lesssim 15$, which was selected during our detection method for the mass estimation of the identified systems. The masses of the systems were found to be consistent with those of the related LH associations as estimated by their MF slopes, giving us confidence in the mass estimation of the detected systems of this survey.

The frequency distribution of the masses found for the intermediate and unbound systems is shown in Fig. 6. It seems to be a normally distributed mass function around a mass of 
Table 4. Sizes of stellar associations in galaxies.

\begin{tabular}{|c|c|c|c|c|c|c|c|}
\hline \multirow{2}{*}{$\begin{array}{l}\text { Galaxy } \\
\text { Name }\end{array}$} & \multirow{2}{*}{$\begin{array}{l}\text { Hubble } \\
\text { Type }\end{array}$} & \multirow[t]{2}{*}{ Number } & \multicolumn{3}{|c|}{ Size $(p c)$} & \multirow[t]{2}{*}{ References } & \multirow{2}{*}{$\begin{array}{c}\text { Detection } \\
\text { Method }\end{array}$} \\
\hline & & & $\min$ & mean & $\max$ & & \\
\hline Sextans A & $\mathrm{E}$ & 3 & & 93 & & Ivanov (1996) & 6 \\
\hline \multirow[t]{2}{*}{ M 31} & $\mathrm{Sb}$ & 210 & 20 & 80 & & Efremov et al. (1987) & 1 \\
\hline & & 15 & & 83 & & Ivanov (1996) & 6 \\
\hline NGC 7331 & $\mathrm{Sb}$ & 142 & & 440 & & Hodge (1986) & 1 \\
\hline \multirow[t]{5}{*}{ M 33} & Sc & 143 & & 200 & & Humphreys \& Sandage (1980) & 1 \\
\hline & & 460 & 30 & 80 & 270 & Ivanov (1987) & 1 \\
\hline & & 289 & 6 & 66 & 305 & Ivanov (1991) & 5 \\
\hline & & 8 & & 87 & & Ivanov (1996) & 6 \\
\hline & & 41 & 10 & 40 & 120 & Wilson (1991) & 3 \\
\hline NGC 2403 & Sc & 88 & 160 & 348 & 600 & Hodge (1985a) & 1 \\
\hline NGC 4303 & $\mathrm{SBbc}$ & 235 & & 290 & & Hodge (1986) & 1 \\
\hline \multirow[t]{3}{*}{ LMC } & Irr & 122 & 15 & 78 & 150 & Lucke \& Hodge (1970) & 1 \\
\hline & & 2883 & 5 & 18 & 272 & Bica et al. (1999) & 1 \\
\hline & & 153 & 21 & 86 & 190 & This Paper & 7 \\
\hline \multirow[t]{3}{*}{ SMC } & Irr & 70 & 18 & 77 & 180 & Hodge (1985b) & 1 \\
\hline & & 31 & 50 & 90 & 270 & Battinelli (1991) & 4 \\
\hline & & $\sim 200$ & 9 & 46 & 234 & Bica \& Schmitt (1995) & 1 \\
\hline \multirow[t]{2}{*}{ NGC 6822} & $\mathrm{Ir}+$ & 16 & 48 & 163 & 305 & Hodge (1977) & 2 \\
\hline & & 6 & & 72 & & Ivanov (1996) & 6 \\
\hline \multirow[t]{2}{*}{ IC 1613} & Irr & 20 & 68 & 164 & 485 & Hodge (1978) & 2 \\
\hline & & 6 & & 83 & & Ivanov (1996) & 6 \\
\hline Pegasus & Irr & 3 & & 65 & & Ivanov (1996) & 6 \\
\hline GR 8 & Irr & 3 & & 114 & & Ivanov (1996) & 6 \\
\hline Ho IX & $\operatorname{Im}$ & 3 & & 72 & & Ivanov (1996) & 6 \\
\hline \multicolumn{8}{|c|}{ DEETECTION METHODS EXPLANATIONS } \\
\hline \multicolumn{8}{|c|}{ 1: Detection by eye on photographic plates or films. } \\
\hline \multicolumn{8}{|c|}{ 2: Detection by eye using star counts from photoelectric and photographic observations. } \\
\hline \multicolumn{8}{|c|}{ 3: "Friends of friends" grouping algorithm on stars from CCD observations. } \\
\hline \multicolumn{8}{|c|}{ 4: "Path Linkage Criterion" applied on O-B2 stars selected from objective-prism observations. } \\
\hline \multicolumn{8}{|c|}{ 5: "Cluster analysis" technique on stars from photographic observations. } \\
\hline \multicolumn{8}{|c|}{ 6: Automated "cluster analysis" technique on OB stars selected from CCD observations. } \\
\hline \multicolumn{8}{|c|}{ 7: Objective statistical method based on star counts from photographic stellar catalogs. } \\
\hline
\end{tabular}

about $10^{3} M_{\odot}$. In this figure the $x$-axis represents the average mass of the systems estimated according to the assumptions of Sect. 4.1 of Paper I and $y$-axis the fraction of systems over the total of intermediate and unbound detected systems (412 in total). We limit this graph at $5 \times 10^{3} M_{\odot}$, which represents the majority $(82 \%)$ of the detected systems. The remaining $18 \%$ of the systems is divided in two groups: The first $9 \%$ includes systems with masses of 5 to $10 \times 10^{3} M_{\odot}$ and the other with masses 10 to $64 \times 10^{3} M_{\odot}$. The distributions of both groups are flat. It is interesting to point out that this distribution implies that the mass function of the stellar associations and open clusters in the LMC seems to be Gaussian.

\subsection{Parameter correlations}

Geyer \& Hopp (1981) presented a correlation of the number of stars and the corresponding system's radius for 12 open and 8 globular clusters in the LMC. They found that the corresponding correlation coefficients are almost the same for both kind of systems. Still, the correlations for these two types of systems are parallel probably due to the systematic differences in number of stars per radius between them. In Fig. 7 (left panel) the original diagram of Geyer \& Hopp (1981) is shown with the corresponding points for the stellar associations found by Kontizas et al. (1994) overplotted. The correlation $r_{\mathrm{h}}\left(N_{\star}\right)$ for these systems is also almost parallel to the correlations of open and globular clusters. In Fig. 7 (right panel) we also present the corresponding correlation of the half-mass radius to the included number of stars for the intermediate and unbound systems of this survey.

In this diagram we observe that there is a differentiation in the radii of the systems at a limit of $r_{\mathrm{h}} \sim 23 \mathrm{pc}$, meaning that there are no intermediate systems with radii larger than this limit, in contrast to the unbound systems. This limit (diameter of $\sim 46 \mathrm{pc}$ ) seems to meet the diameter limit as was defined by Efremov (1982) for clusters and stellar associations. 

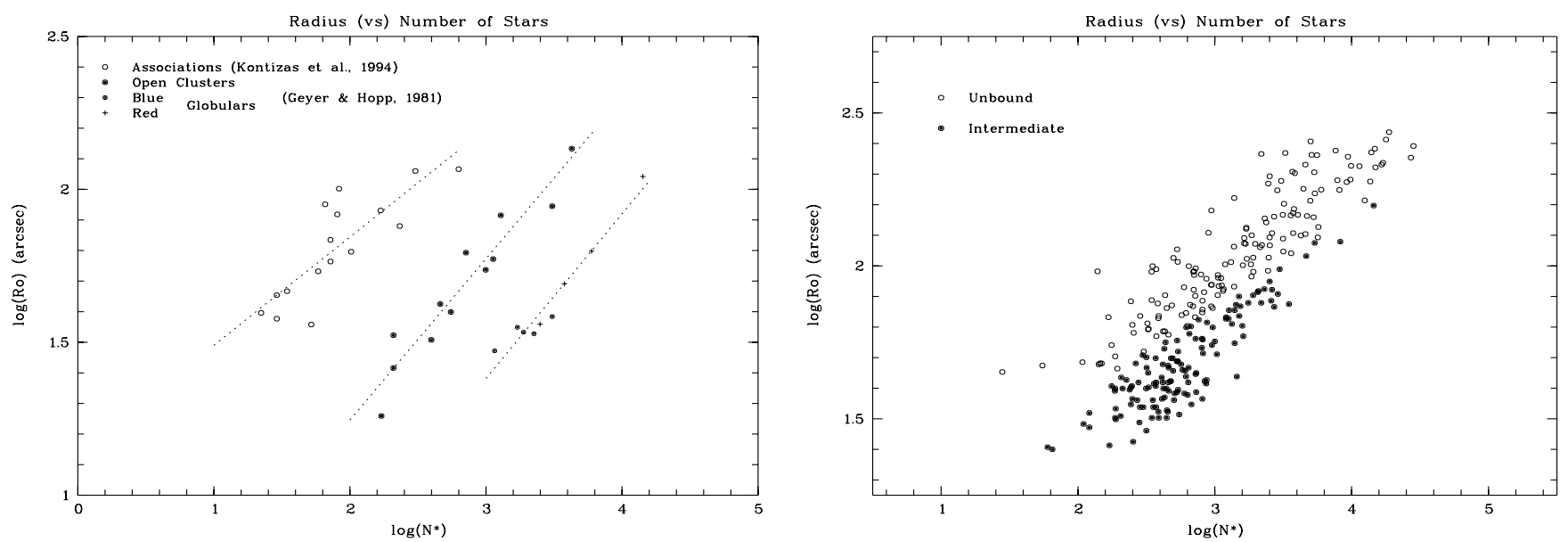

Fig. 7. Correlations of half-mass radii with the corresponding number of stars for stellar associations, open and globular clusters in the LMC (left panel) and for the intermediate and unbound systems of this survey (right panel).

The correlation of number of stars versus radius, as shown in the right panel of Fig. 7 for both classes of systems follows almost an identical trend. Specifically the relation $\log r_{\mathrm{h}}$ (vs.) $\log N_{\star}$ for the intermediate systems has a slope of $0.35 \pm 0.02$, while this slope for the unbound systems is $0.31 \pm 0.01$. Both correlations can be fitted very well to a line. The statistical tests we performed for the goodness of fit, using Spearman and Pearson correlation coefficients, showed that indeed there is a strong correlation between the radius of a system and the corresponding number of stars. The correlation coefficients where found to be between 0.8 and 0.9 .

It is worth noting that these slopes are in a very good agreement with the one for the stellar associations found by Kontizas et al. (1994), which is equal to $\simeq 0.35 \pm 0.06$. On the contrary the slopes for the open and globular clusters of Geyer \& Hopp (1981) are steeper equal to $0.53 \pm 0.05$ and $0.54 \pm 0.05$ correspondingly. We performed additional statistical tests using the Kolmogorov-Smirnov method, in order to detect any possible similarities between the correlations for our systems and the ones of the Kontizas et al. associations and of the Geyer \& Hopp clusters. The only significant similarity found was the one between our intermediate systems and of the open clusters with $96 \%$ of coincidence.

It should be taken under consideration that the parameters used in these correlations are directly measured without any assumption to be used for their estimation. So the number of stars used is the counted stellar number within each system's boundaries from the APM catalog, while $r_{\mathrm{h}}$ is the radius, where half of these stars are contained. Consequently these correlations are physically meaningful, as far as their slopes concerns. Still, they are to be taken arbitrarily due to the systematic incompleteness in the estimation of the actual numbers. Interesting is also the fact that the distributions of associations, open clusters and globulars in the diagram of Geyer \& Hopp (1981) and Kontizas et al. (1994) systems are very well distinct to each other and this is probably due to incomplete samples. The distributions of our larger sample, on the other hand, show that there is no clear distinction between systems of different types, which implies that there might be also "hybrid" systems

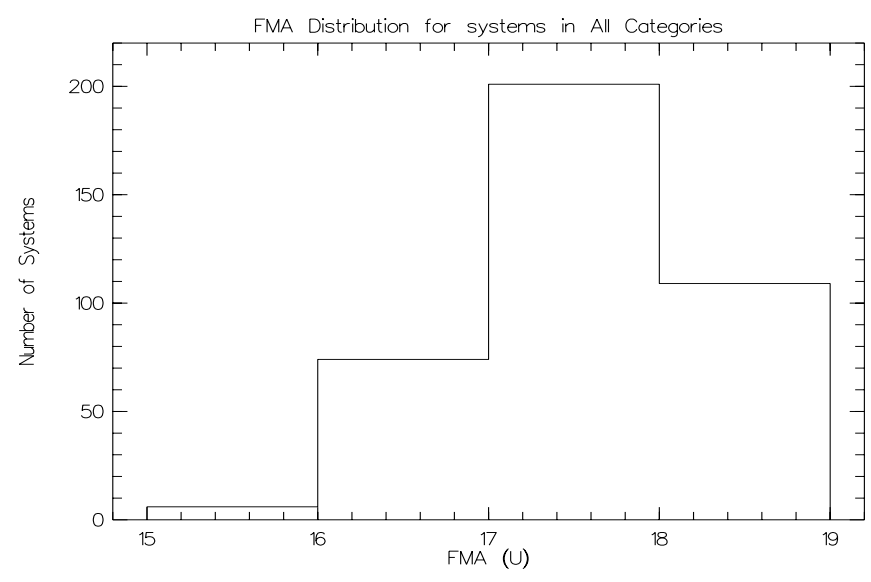

Fig. 8. Number distribution of detected systems in all three categories according to their "Fainter Magnitude of Appearance".

between what we call associations and open clusters as far as these parameters correlations concerns.

\subsection{Fainter magnitude of appearance}

During the detection and study of stellar systems in two selected areas of the $U$ plate, in Paper I, we observed that the fainter magnitude, where each systems first appears in the star counts differs from the one system to the other. We called this limit "Fainter Magnitude of Appearance" (FMA). These differences in the FMA are apparent for systems of the same category, as well as for neighbouring systems. This phenomenon is now verified from the larger sample of systems in this paper. For this investigation we will also use the detected bound systems, since we are only interested in the fainter detected magnitude for every concentration of stars found, without taking any system category under consideration. The number distribution of all the detected systems according to their FMA is given in Fig. 8. In this distribution we use steps of one magnitude in order to achieve statistically significant results, due to the relatively low number of systems. Considering that the detection limit of the plate is at around $U \simeq 20 \mathrm{mag}$, the incompleteness 
around the 19th magnitude can be important. In this case one should expect a larger number of systems in the fainter magnitude bin. Still, the distribution of systems in different FMAs remains.

Interesting seems to be also the spatial distribution of the detected systems according to their FMA. We found that as far as the FMA concerns the systems show to be concentrated in larger structures, most of which have filamentary shape and contains other smaller and denser concentrations of systems. As we note in Paper I this differentiation of the FMA should not depend of the density of the systems, since systems of different types show to have the same FMA. Probably it is also not due to intergalactic absorption, since the LMC reddening is not large enough to produce a magnitude differentiation up to four magnitudes (Harris et al. 1997). In Paper I we give as a possible explanation for this phenomenon the existence of pre-main sequence stars with masses up to $8 M_{\odot}$ in the systems, as was found by various authors for example in R 136 in 30 Doradus (e.g. Sirianni et al. 1999). Still, this is a rough explanation and detailed analysis on selected young stellar systems in the LMC is needed in order to be verified.

\section{Conclusions}

In this paper we present the properties of the stellar associations and open clusters in the LMC, as detected on a digitised, by APM, $1.2 \mathrm{~m}$ UK Schmidt Telescope Plate in $U$, using the method proposed in Paper I. There were found 494 stellar systems in an area of $\sim 6.5 \times 6.5$ around the LMC Bar. We classified the detected systems in three categories based on their stellar density in the half-mass radius, as was estimated for every system according to the method and assumptions of Paper I. There were found 82 bound systems with $\rho \geq 1.0 M_{\odot} \mathrm{pc}^{-3}, 259$ intermediate systems with $0.1 \leq \rho \leq 1.0 M_{\odot} \mathrm{pc}^{-3}$ and 153 unbound systems with $\rho \leq 0.1 M_{\odot} \mathrm{pc}^{-3}$.

Lucke \& Hodge (1970) detected in the same area 102 stellar associations. We detected almost the $70 \%$ of them. The 29 nondetected LH stellar associations are located in dense regions of ionised hydrogen and in the Bar of the galaxy. In these regions the method was unable to detect low concentration systems, due to the incompleteness of the stellar catalog. It was found that the coincidence of the sizes of the LH associations, as were estimated by us with the ones of Lucke \& Hodge is very good. In general we detected about four times more loose young stellar systems than Lucke \& Hodge in their survey of associations. The higher number of identified systems in our survey is probably due to the better detection performance of our method in areas of loose stellar concentrations.

Several studies have demonstrated the coevality of LH stellar associations in their IMF slopes and star formation histories (e.g. Massey 1998; Massey et al. 2000). The selection criteria of our method for the detection of young loose stellar systems in the LMC were based on this coevality concerning their MF slopes and stellar content. In addition, thorough statistical tests were carried out for the best performance of the method in detecting coeval loose concentrations of bright blue stars. These can serve as evidence that the newly discovered unbound and intermediate systems should be considered as candidates for true open clusters and stellar associations and not just statistical coincidences.

The intermediate and unbound systems, as was found from their spatial distribution, seem to construct large filamentary structures. A comparison of the loci of these systems with neutral hydrogen observations shows that filamentary and arc structures are often formed from the concentration of systems on the edges of giant and super-giant HI shells. The intermediate systems cover a size range of about $30 \mathrm{pc}$ up to $160 \mathrm{pc}$. The size distribution of the unbound systems shows two peaks, the higher first at $70 \mathrm{pc}$ and the lower second at $130 \mathrm{pc}$. The average size of these stellar associations ( $86 \mathrm{pc}$ ) is in a very good agreement with the mean size of stellar associations found in various galaxies $(80 \pm 3 \mathrm{pc})$, which possibly represents a global lengthscale of star formation. The mass function of the intermediate and unbound systems was found here to be almost normally distributed around $10^{3} M_{\odot}$.

The correlation of the half-mass radius of every system to the corresponding number of stars, shows the existence of two different system types, the borders of which are overlapping each other. Still, there is a distinction between these two classes at a diameter limit of $\sim 46 \mathrm{pc}$. The differences in the distributions of $r_{\mathrm{h}}$ versus $N_{\star}$ for the intermediate and unbound systems of the survey indicates the different nature of these two kinds of systems, with the unbound representing dynamically loose stellar concentrations, which are expected to dissolve sooner than the intermediate, which seem to represent mostly open clusters.

Throughout the whole catalog of detected systems the Fainter Magnitude of Appearance (FMA) shows variations, which seem to be independent of the system types and of the location of the systems. These variations are found up to four magnitudes, which implies that the LMC absorption may not be the reason and allowing us to suggest the existence of premain sequence stars.

Acknowledgements. The authors would like to express their thanks to the $1.2 \mathrm{~m}$ U.K. Schmidt Telescope Unit for the loan of the plates and the APM \& SuperCOSMOS for the digitisation of them. Our thanks are also due to the General Secretariat of Research and Technology of the Greek Ministry of Development and the British Council for their financial support, as well as to the University of Athens (ELKE). This research has made use of the SIMBAD database, operated at CDS, Strasbourg, France.

\section{References}

Alongi, M., Bertelli, G., Bressan, A., et al. 1993, A\&AS, 97, 851

Battinelli, P. 1991, A\&A, 244, 69

Battinelli, P., \& Efremov, Yu. N. 1999, A\&A, 346, 778

Bhatia, R. K., \& Hatzidimitriou, D. 1988, MNRAS, 230, 215

Bhatia, R. K., \& MacGillivray, H. T. 1989, A\&A, 211, 9

Bhatia, R. K., Read, M. A., Tritton, S., \& Hatzidimitriou, D. 1991, A\&AS, 87, 335

Bica, E. L. D., \& Schmitt, H. R. 1995, ApJS, 101, 41

Bica, E. L. D., Schmitt, H. R., Dutra, C. M., \& Oliveira, H. L. 1999, AJ, 117, 238

Blaauw, A. 1964, ARA\&A, 2, 213

Caldwell, J. A. R., \& Coulson, I. M. 1986, MNRAS, 218, 223

Caldwell, D. A., \& Kutner, M. L. 1996, ApJ, 472, 611

Davies, R. D., Elliot, K. H., \& Meaburn, J. 1976, MmRAS, 81, 89 
de Boer, K. S., Braun, J. M., Vallenari, A., \& Mebold, U. 1998, A\&A, 329, L49

Dohm-Palmer, R. C., Robbie, C., Skillman, E. D., et al. 1997, AJ, 114, 2527

Efremov, Yu. N. 1982, Pis'ma Astr. Zh., 8, 585

Efremov, Yu. N., Ivanov, G. R., \& Nikolov, N. S. 1987, Ap\&SS, 135, 119

Efremov, Yu. N. 1988, Sov. Sci. Rev. E. Astrophys. Space Phys., 7, 105

Efremov, Yu. N., \& Elmegreen, B. G. 1998, MNRAS, 299, 588

Efremov, Yu. N., Elmegreen, B. G., \& Hodge, P. W. 1998, ApJ, 501, 163

Elmegreen, B. G., \& Efremov, Yu. N. 1996, ApJ, 466, 802

Filipovic, M. D., Haynes, R. F., White, G. L., et al. 1995, A\&AS, 111, 311

Garmany, C. D., Massey, Ph., \& Parker, J. W. 1994, AJ, 108, 1256

Geyer, E. H., \& Hopp, U. 1981, in Astroph. Param. Glob. Clust., IAU Coll., 68, 235

Gomez, M., Hartmann, L., Kenyon, S. J., \& Hewett, R. 1993, AJ, 105, 1927

Gouliermis, D., Kontizas, M., Korakitis, R., et al. 2000, AJ, 119, 1737 (Paper I)

Harris, J., Zaritsky, D., \& Thompson, I. 1997, AJ, 114, 1933

Harris, J., \& Zaritsky, D. 1999, AJ, 117, 2831

Henize, K. G. 1956, ApJS, 2, 315

Hodge, P. W., \& Sexton, J. A. 1966, AJ, 71, 363

Hodge, P. W. 1977, ApJS, 33, 69

Hodge, P. W. 1978, ApJS, 37, 145

Hodge, P. W. 1985a, PASP, 97, 530

Hodge, P. W. 1985b, PASP, 97, 1065

Hodge, P. W. 1986, in Luminous Stars and Associations in Galaxies, ed. C. de Loore, A. Willis, \& P. Laskarides (Dordrecht: Reidel), IAU Symp., 116, 369

Hodge, P. W. 1988, PASP, 100, 1051

Humphreys, R. M., \& Sandage, A. R. 1980, ApJS, 44, 319

Hunter, D. A., Elmegreen, B. G., \& Baker, A. 1998, ApJ, 493, 595

Ivanov, G. R. 1987, Ap\&SS, 136, 113

Ivanov, G. R. 1991, Ap\&SS, 178, 227

Ivanov, G. R. 1996, A\&A, 305, 708

Johansson, L. E. B., Greve, A., Booth, R. S., et al. 1998, A\&A, 331, 857

Kim, S., Dopita, M. A., Staveley-Smith, L., \& Bessell, M. 1999, AJ, 118,2797

Kontizas, M., Morgan, D. H., Hadzidimitriou, D., \& Kontizas, E. 1990, A\&AS, 84, 527
Kontizas, M., Kontizas, E., Dapergolas, A., Argyropoulos, S., \& Bellas-Velidis, Y. 1994, A\&AS, 107, 77

Kontizas, E., Kontizas, M., Gouliermis, D., et al. 1999, in New Views of the Magellanic Clouds, ed. Y.-H. Chu, N. Suntzeff, J. Hesser, \& D. Bohlender (San Francisco: ASP), IAU Symp., 190, 410

Kutner, M. L., Rubio, M., Booth, R. S., et al. 1997, A\&AS, 122, 255

Lortet, M. C., \& Testor, G. 1984, A\&A, 139, 330

Lucke, P., \& Hodge, P. W. 1970, AJ, 75, 171

Madore, B. F., \& Freedman, W. L. 1998, ApJ, 492, 110

Maragoudaki, F., Kontizas, M., Kontizas, E., Dapergolas, A., \& Morgan, D. H. 1998, A\&A, 338, L29

Maragoudaki, F., Kontizas, M., Morgan, D. H., et al. 2001, A\&A, 379, 864

Massey, Ph., Garmany, C. D., Silkey, M., \& De Gioia-Eastwood, K. 1989, AJ, 97, 107

Massey, Ph., Johnson, K. E., \& De Gioia-Eastwood, K. 1995, ApJ, 454,151

Massey, Ph. 1998, in The Stellar Initial Mass Function, ed. G. Gilmore, \& D. Howell, (San Francisco: ASP), ASP Conf. Ser., 142, 17

Massey, Ph., Waterhouse, E., \& DeGioia-Eastwood, K. 2000, AJ, 119, 2214

McKibben Nail, V., \& Shapley, H. 1953, Proc. Nat. Acad. Sci, 39, 358

Meaburn, J. 1980, MNRAS, 192, 365

Morgan, D. H. 1992, MNRAS, 258, 639

Nikolaev, S., \& Weinberg, M. D. 2000, ApJ, 542, 804

Oey, M. S., \& Massey, Ph. 1995, ApJ, 452, 210

Parker, J. W. 1993, AJ, 106, 560

Parker, J. W., \& Garmany, C. D. 1993, AJ, 106, 1471

Parker, J. W., Zaritsky, D., Stecher. T. P., Harris, J., \& Massey, P. 2001, AJ, 121, 891

Shapley, H. 1956, Am. Sci., 44, 73

Shapley, H., \& Lindsay, E. M. 1963, IrAJ, 6, 74

Shimmins, A. J., \& Day, G. A. 1968, AuJPh, 21, 377

Sirianni, M., Leitherer, C., Nota, A., \& Clampin, M. 1999, in New Views of the Magellanic Clouds, ed. Y.-H. Chu, N. Suntzeff, J. Hesser, \& D. Bohlender (San Francisco: ASP), IAU Symp., 190, 254

Sulentic, J. W., \& Tifft, W. G. 1973, The revised New General Catalogue of non-stellar objects, Univ. Arizona Press

Westerlund, B. E. 1997, The Magellanic Clouds (Cambridge Univ. Press)

Will, J.-M., Bomans, D. J., \& Dieball, A. 1997, A\&AS, 123, 455

Wilson, C. D. 1991, AJ, 101, 1663

Zaritsky, D., Harris, J., \& Thompson, I. 1997, AJ, 114, 1002 